\title{
The treatment of osteosarcoma in 1992
}

\author{
S.R. Babin ${ }^{1}$ and A. Babin-Boilletot ${ }^{2}$ \\ ' Département d'orthopédie et de traumatologie, ${ }^{2}$ Service d'Onco-Hématologie, Hôpital de Hautepierre, F-67098 Strasbourg Cedex
}

The treatment of osteosarcoma is a team effort in 1992. Dramatic improvements have been made over the last 20 years. The management of the patient includes chemotherapy and surgery, and these two methods are currently inseparable. The chemosensitivity of this tumour has been known since the seventies, when Jaffe tested high-dose methotrexate for the first time. Other cytostatic drugs were found to be efficient such as adriamycin, cyclophosphamide, ifosfamide, and platinum derivates. When given after radical surgery, as it was in 1975, polychemotherapy allows a 5year complete remission rate in the range of $50 \%$. In 1978, Rosen, considering that osteosarcoma presented at diagnosis with micrometastases in $80 \%$ of patients, and having previously shown the efficiency of adjuvant chemotherapy, undertook a trial with neo-adjuvant chemotherapy in New York, administering chemotherapy to the patient before any radical surgery. Histological evaluation of the necrosis found in the tumoral mass after this chemotherapy appeared to be an important prognostic factor: patients were then classified in "good responders" and "bad responders" according to the percentage of tumour necrosis (more or less than 90\%). After surgery, new chemotherapy was given, which was similar to the pre-operative one in the first $T 7$ trial, and adapted to the histological response in the subsequent $\mathrm{T} 10$ trial. The results of these trials, which were brought up to date in 1991 show a 10 year complete remission survival rate near $70 \%$. However the results are worse for "bad responders" than for "good responders". The principle of neo-adjuvant chemotherapy was then accepted and, in 1992, is very commonly used. Its modalities are variable according to different groups; Intra-arterial chemotherapy may also be used, using adriamycin or cisplatinum as done by Jaffe and the Rizzoli group. This method of administration is however debatabie, as the diffusion of drugs, although good in the primary tumour, does not seem to be as efficient in distant metastases. Most groups presently use intra-venous chemotherapy before radical surgery. In addition to the early treatment of micrometastases, neo-adjuvant chemotherapy sometimes leads to better limitation of the tumour and to shrinkage of the soft tissue involvement allowing better surgical conditions. However, late lung metastases do exist, although they are rare, even in patients who were initially "good responders".

Considering local surgery, a biological approach by allograft reconstruction is adopted by many groups; but the complication rate is high, such as infection, nonunion, fracture, instability, or cartilage damage. R.S. Bell, A. Davis et al from Toronto, obtain their best results with intercalary allografts, compared to allograft-arthrodesis, allograft-implant reconstruction and osteo-articular allografts with which the failure rate is about $50 \%$. With osteo-articular allografts on both sides of the knee, M.G. Rock, E.Y.S. Chao et al from the Mayo Clinic estimate the number of failures to be in the range of 40 to $50 \%$ and they think that it is preferable to use a reconstruction pros- thesis in the distal femur. As regards the proximal tibia, they propose using osteo-articular allografts or allograftimplant reconstruction for the reconstitution of the extensor mechanism of the knee. Utilizing custom proximal tibial implants the soft tissue reconstruction consists of the fixation of the remaining patellar tendon into the fascia of the transposed gastrocnemius muscle as proposed by Dubousset and Missenard from Paris (Hôpital Saint-Vincent-dePaul); this means of maintaining extensor capability is a valuable alternative. To improve the fusion between the host bone and the allograft, some authors propose addition of an autologous microvascularised fibular graft to the allograft. R. Cappanna and M. Manfrini, from the Rizzoli Institute in Bologna use this technique for intercalary reconstructions in metaphyseo-diaphysal localisations and their preliminary short term results are encouraging. M.J. O'Connor, M.G. Rock et al from the Mayo Clinic recommand this technique in shoulder arthrodesis, after tumoral resection including the sacrifice of the abductor mechanism. C.M. Clinkscales, M.B. Woodf and H. Sim, from the Mayo Clinic use this principle in the treatment of failures of allograft reconstructions, in case of infection or pseudarthrosis. The pelvic ring reconstruction technique of J. Puget and G. Utheza from Toulouse is interesting because it uses the proximal femur as an autograft: on this femur, one can make an acetabular cavity and fix a megaprosthesis hip. The saddle-prosthesis by E. Nieder from Hamburg, which was initially made for the repla- 
cement of total hip prosthesis with periacetabular defect, can be used after some partial pelvic resections when one decides not to use allograft. The mechanical approach of reconstruction by a metal endoprosthesis raises questions regarding complications related to oncological surgery and to chemotherapy, and questions about the duration of the prosthesis. From an experimental point of view, J. Hua and P.S. Walker from Stanmore show that the strain distribution is better for press-fit stems than for cemented stems however proximal or distal their implantation is. This nearnormal distribution with press-fit stems should decrease the undesirable bone remodeling which is observed at the level of the junction, and improve the longevity of the implant in the bone. Clinically, the follow-up of cemented prostheses is longer than that of pressfit prostheses. P.S. Unwin, J.P. Cobb and P.S. Walker have studied, over a 30 year period, the fate of 221 cemented proximal femoral prostheses and 447 cemented distal femoral prostheses for reconstruction after tumoral resection. The actuarial failure-free survival at 10 years of a proximal femoral prosthesis is $82 \%$ whereas that of a distal femoral prosthesis in $61 \%$. Failure is here defined as the need to reoperate or amputate for reason other than local recurrence.

Despite the obvious progresses, there are yet some questions pending. In addition to the difficult problem of reconstruction after resection in children, in adults the promising solution of the allograft exhibits a high complication rate. Better immunologic knowledge, enhancing vascularisation procedures will perhaps reduce the number of failures. As regards prostheses, their life span is a bet on the future when "press-fit" and modular systems should carry weight. The systemic therapy of the disease with chemotherapy has not yet solved all the problems, such as recurrences or metastases: some patients do not tolerate the standard doses of chemotherapy, and dosages must be adjusted, if possible with an individual pharmacokinetic support. Most of all, some tumors are initially resistant to cytostatic drugs, and those drugs, given in a standard way, are inefficient. In vitro tumor cell studies looking for multidrug resistance genes will perhaps allow the use of efficient drugs as soon as possible. Finally, the study of specific genes and of their amplification could lead in the near future to a better understanding of biological prognostic factors, and to a better adaptation of the treatment of particularly resistant patients.

Bibliographic data are to be found in:

1. Complications of limb salvage; prevention, management and outcome, 6th International Symposium on Limb Salvage, Montreal, September 8-11, 1991. ISOLS, Montreal, 1991

2. Clinical Orthopedics and related research, 270 , September 1991 